\title{
Effect of type 2 diabetes medications on fracture risk
}

\author{
Cristian Guja $^{1,2}$, Loreta Guja ${ }^{2}$, Rucsandra Dănciulescu Miulescu ${ }^{1,2}$ \\ ${ }^{1}$ National Institute of Diabetes, Nutrition and Metabolic Diseases "Prof. N.C. Paulescu”, Bucharest, Romania; “"Carol Davila” University of \\ Medicine and Pharmacy, Bucharest, Romania \\ Contributions: (I) Conception and design: All authors; (II) Administrative support: None; (III) Provision of study materials or patients: None; (IV) \\ Collection and assembly of data: All authors; (V) Data analysis and interpretation: None; (VI) Manuscript writing: All authors; (VII) Final approval of \\ manuscript: All authors. \\ Correspondence to: Assoc. Prof. Cristian Guja. National Institute of Diabetes, Nutrition and Metabolic Diseases "Prof. N.C. Paulescu", 5-7 Ion Movila \\ Street, Bucharest 2, Romania. Email: cristian.guja@b.astral.ro.
}

\begin{abstract}
Type 2 diabetes, one of the most frequent chronic diseases, has an important effect on bone metabolism, with most studies reporting an increased prevalence of fractures in these patients despite an apparently increased bone mineral density. Most probable explanation is an alteration of bone structure/ quality with increased fragility but the different diabetes medications influence the risk of fracture. While metformin and incretin-based therapies are safe, thiazolidinediones and canagliflozin (sodium-glucose cotransporter-2 inhibitor) negatively impact bone metabolism and should be avoided in subjects at increased risk of fractures. Insulin and sulphonylureas are generally safe but can increase the risk of hypoglycemia and falls with subsequent traumatic fractures. Their combination should be avoided, especially in elderly subjects.
\end{abstract}

Keywords: Type 2 diabetes; diabetes drugs; bone; fracture

Submitted Aug 07, 2019. Accepted for publication Sep 05, 2019.

doi: 10.21037/atm.2019.09.51

View this article at: http://dx.doi.org/10.21037/atm.2019.09.51

\section{Introduction}

Diabetes is one of the 4 non-communicable diseases (together with cardiovascular diseases, cancer and pulmonary diseases) established by the World Health Organization (WHO) as the major public health issues of the $21^{\text {st }}$ century. Altogether these 4 explain $71 \%$ of worldwide deaths each year (1). Diabetes, especially type 2 diabetes (T2D), is also one of the most prevalent chronic diseases, the last International Diabetes Federation (IDF) data estimating a total of 425 million subjects affected by this disease in 2017 , representing approximately $8.8 \%$ of the adult population (2). Despite a continuous increase in the life expectancy of T2D subjects (3), diabetes represents an important cause of mortality, with in-between 1.6 million (1) and 4 million (2) deaths annually, i.e., more than deaths by infectious diseases. Diabetes and its chronic complications represent also a major cause of morbidity with huge individual and societal costs. Thus, according to the IDF
2017 Atlas data, the costs associated with diabetes reached 727 billion USD in 2017 (2).

On the other hand, osteoporosis and its direct consequence, osteoporotic fractures, represent another significant cause of morbidity and premature mortality worldwide, with an estimated $\sim 190,000$ deaths annually (4). Moreover, with a continuously aging population, the prevalence of this condition is expected to increase.

During the last years it became widely accepted that diabetes (both T1D and T2D) have an important impact on bone metabolism, with fragility fractures representing an often "neglected" complication of diabetes $(5,6)$. Moreover, with better diabetes care and increased survival of these subjects (7), the prevalence of osteoporosis and fragility fractures in older diabetes patients is expected to increase.

The aim of this review paper is to synthesize the knowledge regarding the effect of different anti-diabetic medications on bone metabolism and fracture risk in T2D patients. 


\section{Diabetes medications, bone metabolism and fracture risk}

\section{Metformin}

Metformin, one of the first used T2D drugs (launched in 1957), is still the cornerstone of T2D therapy, representing the first line treatment currently recommended by international guidelines (8) due to its robust efficacy, low risk of hypoglycemia, positive weight effects and cardiovascular benefits. The exact mechanism of action is not fully elucidated but seems to be mainly mediated by activation of AMPK (AMP activated protein kinase) (9) with subsequent decreased hepatic glucose production and an insulin sensitizing effect.

Experimental and clinical data seem to indicate that metformin has an anabolic/osteogenic action on bone (10), albeit there is controversy on this matter. In addition, there is only scarce published data regarding the effect of metformin on the skeleton and fracture risk in vivo.

In a Danish retrospective case-control study published in 2005 , metformin was shown to significantly reduce the fracture risk (OR 0.81) (11). This was subsequently confirmed by a cohort population-based study performed in Rochester, Minnesota, USA (12) but not in another retrospective French case-control study (13). More recently, a sub-analysis of the TECOS study (testing the CV safety of sitagliptin) showed that metformin treated patients had a reduced fracture risk (OR 0.76) (14).

In conclusion, metformin can be regarded as neutral or maybe beneficial in respect to its bone effects. However, currently there is no dedicated prospective randomized controlled study to analyze the effect of metformin on bone compared to placebo (10).

\section{Sulphonylureas (SUs)}

SUs are insulin secretagogues with potent hypoglycemic action, first used in the 1950's. They stimulate insulin secretion by the pancreatic beta cells following binding to specific receptors (SUR component of the K-ATP channel) (8). They associate rather high hypoglycemic risk and reduced durability of the metabolic effect in time (15) but were associated with reduced prevalence of diabetes microvascular complications in the UKPDS study (8).

By increasing insulin levels, SUs are expected to have a positive impact on bone metabolism. Some early studies indicated that glimepiride enhances proliferation of osteoblasts and their osteogenic differentiation in an experimental animal model (16). However, human studies showed no effect of SUs on markers of bone resorbtion/ formation neither on measures of bone mineral density (BMD) $(17,18)$.

There are relatively few studies that reported the effect of SUs on fracture risk in T2D patients. One of the first was the ADOPT trial that originally aimed to evaluate the durability of hypoglycemic effect of rosiglitazone, metformin and glyburide (SU) (19). The study showed that patients treated with metformin and $\mathrm{SU}$ had a similar rate of fractures $(7.3 \%$ vs. $7.7 \%$ over 5 years) but lower than rosiglitazone. The data were confirmed by the studies of Vestergard in Denmark (OR 0.82) (11) and Melton in Rochester USA (HR 0.9) (12). However, other studies indicate an increased fracture risk. For example, in the multinational MrOS (Osteoporotic Fractures in Men) study that enrolled 5,994 men, SU treatment increased the risk for fractures with $66 \%$ (HR 1.66) (20). Similarly, the analysis of a registry-based cohort of US T2D patients, indicated an increased rate of hip fractures (1.7\% vs. $1.2 \%$, OR 1.46) in older man and women (21). Overall, a metaanalysis published in 2013 indicated no correlation between SU use and risk of fall and fractures in T2D patients (22).

In conclusion, SUs seem not to directly increase fracture risk in T2D subjects. However, this could be possible by increasing the risk of falls secondary to hypoglycemia, more often in older, frail subjects and especially in the case of associated insulin use (23).

\section{Thiazolidinediones}

Thiazolidinediones (TZDs) are selective agonists of the nuclear receptors of the PPAR $\gamma$ family. They influence expression of multiple genes involved in glucose and energy metabolism, especially in the adipose tissue, liver and muscles. The net effect is increased insulin sensitivity with improved metabolic control and a significant decrease of HbA1c in insulin resistant T2D patients (24). Despite the metabolic benefits, TZD treatment is usually associated with weight increase explained by accumulation of adipose tissue, especially in the subcutaneous compartment, but concomitant decrease of liver fat and visceral adipose tissue. Their adverse effect profile includes also increased risk of hospitalization for heart failure and increased risk of fractures (24).

There are several experimental animal and preclinical data indicating that TZDs might have deleterious effects on bone structure (25). Thus TZDs (both rosiglitazone 
and pioglitazone) seem to increase osteoclast formation and decrease osteoblast formation (following the preferentially differentiation of mesenchymal stem cells into adipogenic cells and not osteoblasts) in mice $(26,27)$. Consequently, TZDs decrease BMD and impair bone micro-architecture (28). These data were confirmed also by human studies, with a recent meta-analysis indicating a significant decrease of $\mathrm{BMD}$ at the lumbar spine, total hip and forearm (but no effect at femoral neck), an effect that was not reversible, even after one year after TZD treatment stop (25).

The adverse effects of TZDs on bone in humans were already reported by the ADOPT trial as early as 2006 $(17,19)$. Thus, results showed an increased risk of upper and lower limb fractures in women treated with rosiglitazone compared to those receiving metformin (HR 1.81) or glyburide (HR 2.13). The effect was particularly strong in menopausal women but could not be established in men (19). The apparent detrimental effect of TZDs in women but not in men was subsequently reconfirmed in a large retrospective study from the USA (more than 19,000 subjects included) (29). In this analysis, the fracture risk was increased with 57\% in women using TZDs versus any other diabetes treatment, with the highest risk after the age of 65 years (HR 1.72). No effect was identified in men (HR 1.05) (29).

Over time data accumulated and now there are available three meta-analyses of studies investigating this association. The first was published by Loke et al. in 2009 and included 10 randomized controlled trials (RCTs) and 2 observational studies (overall more than 45,000 analyzed subjects) (30). The 10 RCTs showed a significantly increased risk of fractures overall for both rosiglitazone and pioglitazone (OR 1.45), with five RCTs confirming the association in women but not in men. The second was published by Bazelier et al. in 2013 and included 3 population based retrospective registry studies from the UK, Holland and Denmark (31). The combined analysis showed a $40 \%$ increased risk of fractures (upper and lower limbs but not hip/femur or vertebral) with TZDs versus any other diabetes medication in women (HR 1.44) but no effect in men (HR 1.05). The largest meta-analysis to date was published in 2014 by Zhu et al. and included 22 RCTs with $\sim 25,000$ subjects and a follow-up period of around 2 years (32). This analysis indicated an almost double risk of fracture with TZD treatment in women (OR 1.94, $\mathrm{P}<0.001$ ) and again, no effect in men (OR 1.02). The association was confirmed both for rosiglitazone (OR 2.01) and pioglitazone (OR 1.73). There is yet no biological explanation for the different effect of TZDs in women compared to men $(33,34)$.
All these meta-analyses indicated that the increased risk of fracture appears after one year of TZD treatment (33). There is only limited information regarding the duration of the negative effect of TZDs on bones after treatment discontinuation. As stated above, a meta-analysis of 5 studies indicated that the BMD decrease persists one year after treatment stop (25). However, a sub-analysis of data from the ACCORD trial indicated that risk of fractures decreases in time after TZD discontinuation, with the risk being similar with those of women never using the drugs after around 2 years (35).

In conclusion, TZD treatment has a negative effect on bone in humans and is associated with increased fracture risk in women, especially after menopause, but not in men.

\section{Dipeptidyl peptidase 4 inbibitors (DPP4i)}

DPP4i represent a modern class of diabetes medications that improve glucose levels by increasing glucagon like peptide 1 (GLP-1) levels to physiological levels (36). They are extensively used in the treatment of T2D, despite their moderate efficacy, due to several other advantages (oral administration, single daily dose, minimal risk of hypoglycemia, weight neutrality and cardiovascular safety) (36).

Preclinical experimental data seem to indicate a positive effect of DPP4i on bone metabolism. Thus, these drugs seem to reduce the bone resorption activity of osteoclasts and promote bone anabolism, with final benefit on bone morphology (37). Consequently, several studies with high doses of sitagliptin in ovariectomized mice/rats (38) indicated an increased vertebral BMD. However, other studies did not confirm a benefit of DPP4i treatment so that a recent review (39) concludes that data on this matter are contradictory and not conclusive.

Regarding human clinical data, results of long-term DPP4 $\mathrm{i}$ use seem to indicate a neutral effect on BMD and risk of fractures $(34,40)$. This is despite an early metaanalysis of 28 RCTs (41) that seemed to indicate a reduced risk of fractures (OR of 0.6). The positive effect was confirmed by a retrospective analysis of T2D patients from primary practices in Germany (42) which matched 4,160 DPP4i ever users to non-users. The study evidenced a reduced risk of fractures (HR of 0.67 ) both in women and men. However, these data were not reconfirmed by a subsequent retrospective population cohort analysis of patients from the UK Clinical Practice Research Datalink (43). The study showed that even after more than 4 years of DPP4i use, the risk of any fracture was 
similar with that of other diabetes medications (HR 0.99). Neutrality of DPP4i was shown also by a metaanalysis including $51 \mathrm{RCTs}, 37$ comparing DPP4i with placebo and 14 DPP4i with another diabetes drug (44). Similar results were provided by the analysis of fracture frequency in the large scale $\mathrm{CV}$ outcome trials with saxagliptin (SAVOR-TIMI 54) (45) and sitagliptin (TECOS) (14), showing the safety (but also lack of benefit) of DPP4 $i$ treatment regarding the risk of fractures: HR of 1.0 with saxagliptin and 1.01 with sitagliptin. Finally, the largest to-date reported meta-analysis included 62 RCTs and more than 62,000 participants (including $~ 33,500$ treated with a DPP4i) (46). With an overall number of 722 analyzed fracture events, this study showed the neutrality of DPP4i treatment $(\mathrm{RR}=0.95, \mathrm{P}=0.5)$.

In conclusion, available data confirmed the safety of DPP4i treatment for the bone. Some studies (metaanalyses and retrospective cohort data) suggested a reduced risk of fracture but these data are controversial. Further well designed RCTs with a longer follow-up duration are required to elucidate this matter.

\section{Glucagon like peptide 1 agonists (GLP-1RAs)}

GLP-1RAs decrease blood glucose levels by promoting insulin secretion and decreasing glucagon secretion, both in a glucose dependent manner, with a minimal risk of hypoglycemia. In addition they promote weight loss, and decrease blood pressure, with a favorable $\mathrm{CV}$ safety profile and even $\mathrm{CV}$ protection for some molecules of this class (47).

GLP-1 receptors are expressed on bone cells (osteoblasts and osteoclasts) and administration of GLP-1RAs in animal models seem to increase bone mass (especially trabecular bone but not cortical bone) in ovariectomised mice (48) and rats (49) for both exenatide and liraglutide. Another study with exenatide in $\mathrm{T} 2 \mathrm{D}$ rats showed an increase in femoral BMD (50). These experimental animal results were not reconfirmed by clinical human data, with an exenatide study showing no effect on BMD despite weight loss (51). Authors interpreted this as a positive result since weight loss was expected to promote BMD decrease and this was blunted by the GLP-1RA administration.

In respect to GLP-1RA treatment effect on fracture risk, a nationwide Danish case-control study showed that current GLP-1RA use is not associated with the risk of fractures (52). In 2014 Mabilleau and colleagues published a meta-analysis of 28 RCTs with GLP-1RAs, of which only 7 reported the occurrence of fractures, with a total number of 19 events (53).
Overall, the analysis indicated that this class does not increase the risk of fractures (OR 0.75, $\mathrm{P}=0.57$ ). A more recent meta-analysis included 16 RCTs with GLP-1RAs with a total of more than 11,000 subjects and 38 fractures (54). Overall, again there was no increased risk of fractures with this class of diabetes drugs (OR 1.05) but differences were noted between liraglutide (decreased risk of fracture) and exenatide (increased risk of fracture).

In conclusion, there is only limited evidence regarding the effect of GLP-1RAs on bone in humans, with current data indicating safety of these class of drugs but, probably, no clinical benefit.

\section{Sodium-glucose cotransporter-2 inbibitors (SGLT2i)}

SGLT2is are the latest class of oral antidiabetic drugs for the treatment of T2D (8). They block the resorption of glucose and natrium in the proximal convoluted tubules inducing glycosuria, with the net balance of approximately $70 \mathrm{~g}$ of glucose lost each day. Beyond improved metabolic control, SGLT2i lead to weight loss, decreased BP values and a positive effect on CV risk (8). Results of large scale RCTs investigating the effect of SGLT2 is on CV risk (EMPA-REG and CANVAS) also indicated a cardio and nephroprotective effect for these drugs (55).

Several physiological and preclinical data indicate that SGLT2i might have a negative influence on bone metabolism. Thus, it was reported that these drugs might increase phosphorus in the proximal convoluted tubules with a slight increase in levels of phosphorus in the serum (56), increase that is higher in subjects with chronic kidney disease (CKD) (57). In the same time, canagliflozin was shown to increase plasma levels of fibroblast growth factor 23 (FGF23), with subsequent decrease of active vitamin D $\left[1,25(\mathrm{OH})_{2} \mathrm{D}\right]$ levels and increase in parathormone (57), a mechanism that was previously reported in a mouse animal model (58). All these changes could promote bone loss. In addition, SGLT2i induce natriuresis with consequent decreased serum natrium that could activate osteoclasts (59). SGLT2i also promote weight loss, reported to be associated with bone resorption and might induce orthostatic hypotension with increased risk of falls (60). Canagliflozin (61) but not dapagliflozin (62) were reported to decrease BMD in long term (up to 104 weeks) treatment of T2D patients.

In respect to the effect of SGLT2i on fracture risk, the first report of an increased risk came from a 104-week study with dapagliflozin in subjects with T2D and moderate CKD (known to have detrimental effects on bone) (63). In 
this study there was a numerical imbalance in the number of fractures, with 13 cases (9.4\%) in the dapagliflozin treated patients and none in the placebo group. Other studies in T2D subjects without CKD did not confirm this detrimental effect. For example, a recent population-based observational study compared 4,548 T2D subjects initiated with dapagliflozin treatment and 18,070 patients initiated with other T2D medication. After an observation of 3 years, there was no difference in the risk of any fractures (HR $=0.89, \mathrm{P}=0.427$ ) (64).

In the large-scale CANVAS program (evaluating the $\mathrm{CV}$ safety of canagliflozin) were included 10.142 T2D subjects with high CV risk that were followed for a median of 126 weeks (65). In this study, canagliflozin treated patients had 26\% increased risk of all fractures (HR 1.26, 95\% CI: 1.04 to 1.52$)$. In contrast, fracture risk was similar for empagliflozin compared to placebo $(3.9 \%$ vs. $3.8 \%)$ in the large scale EMPA-REG Outcome trial (7,020 T2D patients with established $\mathrm{CV}$ disease followed-up for a median of 3.1 years) (66). More recently, the safety of empagliflozin was confirmed by a combined analysis of over 12,000 subjects from phase I-III RCTs and a direct comparison with glimepiride (67). Finally, a recent meta-analysis included 20 RCTs with SGLT2i with a total of 8,286 subjects and showed no increased risk of fracture with these drugs in the combined analysis ( $\mathrm{RR}=0.67,95 \% \mathrm{CI}: 0.37$ to $1.07)$ and also for the individual analysis of canagliflozin, dapagliflozin and empagliflozin (68).

In conclusion, despite several physiological data regarding a possible detrimental effect of SGLT2i for the bone, clinical evidence in support of this hypothesis is limited to data with canagliflozin in the CANVAS program. No increased risk could be evidenced for dapagliflozin and empagliflozin. Of note, canagliflozin also inhibits the function of SGLT1 with larger expression in the gut. Further studies are required to fully elucidate the bone effects of these T2D medications.

\section{Insulin}

Discovered by Nicolae Paulescu in 1921 and first used for the treatment of diabetes in humans in 1922 by Frederick Banting, insulin is the oldest diabetes drug and still the most efficient (8), with practically unlimited hypoglycaemic potency. In the same time insulin is a potent anabolic hormone, with important influences on bone metabolism (69).

Experimental preclinical animal studies showed that insulin might improve BMD following signalling via IGF-
1 and AMPK activation (70,71). However, human data are somehow controversial. Although some report no negative effect of insulin on bone microarchitecture in humans (40), a more recent study reported that insulin use in older T2D subjects is negatively associated with BMD, bone microarchitecture and bone strength of the distal radius (72). The authors admit that, despite adjusting for HbAlc and disease duration, residual confounding factors might explain this negative result.

Regarding the effect of insulin treatment on fracture risk, most studies report an increased risk $(34,40)$. Thus, an early study by Schwartz et al. (Study of Osteoporotic Fractures) included 9,654 older ( $>65$ years) women, of which 106 were insulin treated (73). After a median followup of 3.7-9.4 years, insulin treatment almost doubled the risk of foot fractures (RR 2.66). The risk is not confined to women, as shown by the multinational MrOS study (20). Thus, the analysis of 5,994 men (age $>65$ years) of which 80 were insulin-treated showed a similarly increased risk (RR 2.46 for non-vertebral fractures) that was maintained after adjustment for multiple variables. Similar data were obtained in the population-based cohort study from Rochester, Minnesota, USA, reporting a 30\% increased risk of fractures for insulin-treated T2D subjects (both men and women) compared to other glucose lowering medications (12). Contrasting with all the above, the Danish case-control study of Vestergaard et al. indicated that insulin treatment does not increase the risk of fractures, reporting instead a non-significant trend towards decreased risk (11). A recent meta-analysis by Moayeri and colleagues showed that insulin treatment is associated with increased fracture risk in T2D subjects, with an overall summary relative risk of 1.52 (74).

There is controversy if the reported increased risk with insulin is a direct effect of the last or only an indirect association. Even though current diabetes guidelines allow the use of insulin for the treatment of T2D subjects already from the second tier after failure of metformin monotherapy (8), insulin is often initiated very late during the course of the disease (75). Thus, one can speculate that insulin treatment might represent an indirect marker of an older, frail, population, frequently with longer diabetes duration and more advanced diabetes complications and, consequently, with increased fracture risk (34). In addition, insulin treatment is reported to increase the risk of hypoglycaemia and falls, consequently increasing the risk of fractures (76). As a proof for this hypothesis, treatment with lower hypoglycaemia risk basal insulin analogues seem to decrease the risk of fracture compared to human NPH insulin (77). 
In conclusion, despite majority of preclinical studies reporting a positive anabolic effect of insulin on bone metabolism, clinical data most often report an increased risk of fracture for insulin treated subjects. However, no definite conclusion can be drawn at this time if this is a direct effect of insulin treatment or rather an indirect association explained by the increased risk of hypoglycaemia and falls characteristic for these subjects. As for other diabetes drugs, properly designed and powered prospective RCTs are required to elucidate this issue.

\section{Conclusions}

T2D subjects are exposed to increased risk of fragility fractures, despite apparently increased BMD. It is also evident that medications used for the treatment of T2D might influence this risk. Available data indicate that TZDs definitely increase the fracture risk (especially in postmenopausal women) and should be avoided in subjects with a high risk of fractures. Caution should be exercised also for the use of canagliflozin (and maybe other SGLT2i) in these subjects. There is absolute safety for the incretin based therapies (DPP4i and GLP-1RAs). Insulin and sulphonylureas seem to be safe in respect to their effects on bone metabolism. However, by increasing the risk of hypoglycemia and traumatic falls, they can increase the risk of fractures especially in elderly, frail subjects. The association of the two should be avoided. Finally, metformin is safe and might even confer some benefits.

Achievement of optimal glycemic control while minimizing the risk of hypoglycemia and avoiding medications with proven negative effect on bone metabolism, combined with osteoporosis treatment remains the cornerstone for preventing fractures in T2D subjects.

\section{Acknowledgments}

None.

\section{Footnote}

Conflicts of Interest: C Guja participated in scientific advisory boards and received consulting fees from AstraZeneca, Bayer AG, Boheringer Ingelheim, Berlin-Chemie Mennarini, Egis, Eli Lilly, Merck KGaA, Merck Sharp \& Dohme, Novo Nordisk, and Sanofi. Other authors have no conflicts of interest to declare.
Ethical Statement: The authors are accountable for all aspects of the work in ensuring that questions related to the accuracy or integrity of any part of the work are appropriately investigated and resolved.

\section{References}

1. World Health Organization. Noncommunicable diseases. Key facts. WHO Factsheet 1 June 2018. Available online: http://www.who.int/news-room/fact-sheets/detail/ noncommunicable-diseases on 18 Jul 2018.

2. International Diabetes Federation. IDF Diabetes Atlas 8th Edition. Available online: http://www.diabetesatlas. org/ on 2 Dec 2017

3. Ioacara S, Guja C, Ionescu-Tirgoviste C, et al. Improvements in life expectancy in adult type 2 diabetes patients in the last six decades. Diabetes Res Clin Pract 2011;92:400-4.

4. Sànchez-Riera L, Wilson N. Fragility fractures \& their impact on older people. Best Pract Res Clin Rheumatol 2017;31:169-91.

5. Poiană C, Capatina C. Fracture risk assessment in patients with diabetes mellitus. J Clin Densitom 2017;20:432-43.

6. Napoli N, Chandran M, Pierroz DD, et al. Mechanisms of diabetes mellitus-induced bone fragility. Nat Rev Endocrinol 2017;13:208-19.

7. Rawshani A, Rawshani A, Franzén S, et al. Mortality and cardiovascular disease in type 1 and type 2 diabetes. N Engl J Med 2017;376:1407-18.

8. American Diabetes Association. Pharmacologic approaches to glycemic treatment, Sec. 8 in Standards of Medical Care in Diabetes-2018. Diabetes Care 2018;41:S73-85.

9. Rena G, Hardie DG, Pearson ER. The mechanisms of action of metformin. Diabetologia 2017;60:1577-85.

10. McCarthy AD, Cortizo AM, Sedlinsky C. Metformin revisited: Does this regulator of AMP-activated protein kinase secondarily affect bone metabolism and prevent diabetic osteopathy. World J Diabetes 2016;7:122-33.

11. Vestergaard P, Rejnmark L, Mosekilde L. Relative fracture risk in patients with diabetes mellitus, and the impact of insulin and oral antidiabetic medication on relative fracture risk. Diabetologia 2005;48:1292-9.

12. Melton LJ, Leibson CL, Achenbach SJ, et al. Fracture risk in type 2 diabetes: update of a population-based study. J Bone Miner Res 2008;23:1334-42.

13. Monami M, Cresci B, Colombini A, et al. Bone fractures and hypoglycemic treatment in type 2 diabetic patients: a 
case-control study. Diabetes Care 2008;31:199-203.

14. Josse RG, Majumdar S, Zheng Y, et al. Sitagliptin and risk of fractures in type 2 diabetes: Results from the TECOS trial. Diabetes Obes Metab 2017;19:78-86.

15. Kahn SE, Haffner SM, Heise MA, et al. Glycemic durability of rosiglitazone, metformin, or glyburide monotherapy. N Engl J Med 2006;355:2427-43.

16. Ma P, Gu B, Xiong W, et al. Glimepiride promotes osteogenic differentiation in rat osteoblasts via the PI3K/ Akt/eNOS pathway in a high glucose microenvironment. PLoS One 2014;9:e112243.

17. Zinman B, Haffner SM, Herman WH, et al. Effect of rosiglitazone, metformin, and glyburide on bone biomarkers in patients with type 2 diabetes. J Clin Endocrinol Metab 2010;95:134-42.

18. Vianna AGD, de Lacerda CS, Pechmann LM, et al. Vildagliptin has the same safety profile as a sulfonylurea on bone metabolism and bone mineral density in postmenopausal women with type 2 diabetes: a randomized controlled trial. Diabetol Metab Syndr 2017;9:35.

19. Kahn SE, Zinman B, Lachin JM, et al. Rosiglitazoneassociated fractures in type 2 diabetes: an analysis from A Diabetes Outcome Progression Trial (ADOPT). Diabetes Care 2008;31:845-51.

20. Napoli N, Strotmeyer ES, Ensrud KE, et al. Fracture risk in diabetic elderly men: the MrOS study. Diabetologia 2014;57:2057-65.

21. Rajpathak SN, Fu C, Brodovicz KG, et al. Sulfonylurea use and risk of hip fractures among elderly men and women with type 2 diabetes. Drugs Aging 2015;32:321-7.

22. Lapane KL, Yang S, Brown MJ, et al. Sulfonylureas and risk of falls and fractures: a systematic review. Drugs Aging 2013;30:527-47.

23. Lapane KL, Jesdale BM, Dubé CE, et al. Sulfonylureas and risk of falls and fractures among nursing home residents with type 2 diabetes mellitus. Diabetes Res Clin Pract 2015;109:411-9.

24. Nanjan MJ, Mohammed M, Prashantha Kumar BR, et al. Thiazolidinediones as antidiabetic agents: A critical review. Bioorg Chem 2018;77:548-67.

25. Billington EO, Grey A, Bolland MJ. The effect of thiazolidinediones on bone mineral density and bone turnover: systematic review and meta-analysis. Diabetologia 2015;58:2238-46.

26. Wan Y, Chong LW, Evans RM. PPAR-gamma regulates osteoclastogenesis in mice. Nat Med 2007;13:1496-503.

27. Smith SY, Samadfam R, Chouinard L, et al. Effects of pioglitazone and fenofibrate co-administration on bone biomechanics and histomorphometry in ovariectomized rats. J Bone Miner Metab 2015;33:625-41.

28. Lazarenko OP, Rzonca SO, Hogue WR, et al. Rosiglitazone induces decreases in bone mass and strength that are reminiscent of aged bone. Endocrinology 2007;148:2669-80.

29. Habib ZA, Havstad SL, Wells K, et al. Thiazolidinedione use and the longitudinal risk of fractures in patients with type 2 diabetes mellitus. J Clin Endocrinol Metab 2010;95:592-600.

30. Loke YK, Singh S, Furberg CD. Long-term use of thiazolidinediones and fractures in type 2 diabetes: a metaanalysis. CMAJ 2009;180:32-9.

31. Bazelier MT, de Vries F, Vestergaard P, et al. Risk of fracture with thiazolidinediones: an individual patient data meta-analysis. Front Endocrinol (Lausanne) 2013;4:11.

32. Zhu Z-N, Jiang Y-F, Ding T. Risk of fracture with thiazolidinediones: an updated metaanalysis of randomized clinical trials. Bone 2014;68:115-23.

33. Wolverton D, Blair MM. Fracture risk associated with common medications used in treating type 2 diabetes mellitus. Am J Health Syst Pharm 2017;74:1143-51.

34. Schwartz AV. Diabetes, bone and glucose-lowering agents: clinical outcomes. Diabetologia 2017;60:1170-9.

35. Schwartz AV, Chen H, Ambrosius WT, et al. Effects of TZD use and discontinuation on fracture rates in ACCORD Bone Study. J Clin Endocrinol Metab 2015;100:4059-66.

36. Zhong J, Gong Q, Goud A, et al. Recent advances in dipeptidyl-peptidase-4 inhibition therapy: lessons from the bench and clinical trials. J Diabetes Res 2015;2015:606031.

37. Glorie L, Behets G, Baerts L, et al. DPP IV inhibitor treatment attenuates bone loss and improves mechanical bone strength in male diabetic rats. Am J Physiol Endocrinol Metab 2014;307:E447-55.

38. Cusick T, Mu J, Pennypacker B, et al. Bone loss in the oestrogen-depleted rat is not exacerbated by sitagliptin, either alone or in combination with a thiazolidinedione. Diabetes Obes Metab 2013;15:954-7.

39. Lecka-Czernik B. Diabetes, bone and glucose-lowering agents: basic biology. Diabetologia 2017;60:1163-9.

40. Kheniser KG, Polanco Santos CM, Kashyap SR. The effects of diabetes therapy on bone: A clinical perspective. J Diabetes Complications 2018;32:713-9.

41. Monami M, Dicembrini I, Antenore A, et al. Dipeptidyl peptidase-4 inhibitors and bone fractures: a metaanalysis of randomized clinical trials. Diabetes Care 2011;34:2474-6. 
42. Dombrowski S, Kostev K, Jacob L. Use of dipeptidyl peptidase-4 inhibitors and risk of bone fracture in patients with type 2 diabetes in Germany-A retrospective analysis of real-world data. Osteoporos Int 2017;28:2421-8.

43. Driessen JH, van den Bergh JP, van Onzenoort HA, et al. Long-term use of dipeptidyl peptidase-4 inhibitors and risk of fracture: A retrospective population-based cohort study. Diabetes Obes Metab 2017;19:421-8.

44. Mamza J, Marlin C, Wang C, et al. DPP-4 inhibitor therapy and bone fractures in people with Type 2 diabetes - A systematic review and meta-analysis. Diabetes Res Clin Pract 2016;116:288-98.

45. Mosenzon O, Wei C, Davidson J, et al. Incidence of fractures in patients with type 2 diabetes in the SAVORTIMI 53 Trial. Diabetes Care 2015;38:2142-50.

46. Fu J, Zhu J, Hao Y, et al. Dipeptidyl peptidase-4 inhibitors and fracture risk: an updated meta-analysis of randomized clinical trials. Sci Rep 2016;6:29104.

47. Htike ZZ, Zaccardi F, Papamargaritis D, et al. Efficacy and safety of glucagon-like peptide-1 receptor agonists in type 2 diabetes: A systematic review and mixedtreatment comparison analysis. Diabetes Obes Metab 2017;19:524-36.

48. Pereira M, Jeyabalan J, Jørgensen CS, et al. Chronic administration of glucagon-like peptide-1 receptor agonists improves trabecular bone mass and architecture in ovariectomised mice. Bone 2015;81:459-67.

49. Lu N, Sun H, Yu J, et al. Glucagon-like peptide-1 receptor agonist liraglutide has anabolic bone effects in ovariectomized rats without diabetes. PLoS One 2015;10:e0132744.

50. Kim JY, Lee SK, Jo KJ, et al. Exendin-4 increases bone mineral density in type 2 diabetic OLETF rats potentially through the down-regulation of SOST/sclerostin in osteocytes. Life Sci 2013;92:533-40.

51. Bunck MC, Eliasson B, Cornér A, et al. Exenatide treatment did not affect bone mineral density despite body weight reduction in patients with type 2 diabetes. Diabetes Obes Metab 2011;13:374-7.

52. Driessen JH, van Onzenoort HA, Starup-Linde J, et al. Use of Glucagon-Like-Peptide 1 receptor agonists and risk of fracture as compared to use of other anti-hyperglycemic drugs. Calcif Tissue Int 2015;97:506-15.

53. Mabilleau G, Mieczkowska A, Chappard D. Use of glucagon-like peptide-1 receptor agonists and bone fractures: a meta-analysis of randomized clinical trials. J Diabetes 2014;6:260-6.

54. Su B, Sheng H, Zhang M, et al. Risk of bone fractures associated with glucagon-like peptide-1 receptor agonists' treatment: a meta-analysis of randomized controlled trials. Endocrine 2015;48:107-15.

55. Hallow KM, Greasley PJ, Helmlinger G, et al. Evaluation of renal and cardiovascular protection mechanisms of SGLT2 inhibitors: model-based analysis of clinical data. Am J Physiol Renal Physiol, 2018;315:F1295-306.

56. Nauck MA, Del Prato S, Meier JJ, et al. Dapagliflozin versus glipizide as add-on therapy in patients with type 2 diabetes who have inadequate glycemic control with metformin: a randomized, 52-week, double-blind, active controlled noninferiority trial. Diabetes Care 2011;34:2015-22.

57. Blau JE, Bauman V, Conway EM, et al. Canagliflozin triggers the FGF23/1,25-dihydroxyvitamin D/PTH axis in healthy volunteers in a randomized crossover study. JCI Insight 2018;3. doi: 10.1172/jci.insight.99123.

58. Thrailkill KM, Clay Bunn R, Nyman JS, et al. SGLT2 inhibitor therapy improves blood glucose but does not prevent diabetic bone disease in diabetic DBA/2J male mice. Bone 2016;82:101-7.

59. Ayus JC, Negri AL, Kalantar-Zadeh K, et al. Is chronic hyponatremia a novel risk factor for hip fracture in the elderly? Nephrol Dial Transplant 2012;27:3725-31.

60. Blau JE, Taylor SI. Adverse effects of SGLT2 inhibitors on bone health. Nat Rev Nephrol 2018;14:473-4.

61. Bode B, Stenlöf K, Harris S, et al. Long-term efficacy and safety of canagliflozin over 104 weeks in patients aged 55-80 years with type 2 diabetes. Diabetes Obes Metab 2015;17:294-303.

62. Ljunggren Ö, Bolinder J, Johansson L, et al. Dapagliflozin has no effect on markers of bone formation and resorption or bone mineral density in patients with inadequately controlled type 2 diabetes mellitus on metformin. Diabetes Obes Metab 2012;14:990-9.

63. Kohan DE, Fioretto P, Tang W, et al. Long-term study of patients with type 2 diabetes and moderate renal impairment shows that dapagliflozin reduces weight and blood pressure but does not improve glycemic control. Kidney Int 2014;85:962-71.

64. Toulis KA, Bilezikian JP, Thomas GN, et al. Initiation of dapagliflozin and treatment-emergent fractures. Diabetes Obes Metab 2018;20:1070-4.

65. Neal B, Perkovic V, Mahaffey KW, et al. Canagliflozin and cardiovascular and renal events in type 2 diabetes. N Engl J Med 2017;377:644-57.

66. Zinman B, Wanner C, Lachin JM, et al. Empagliflozin, cardiovascular outcomes, and mortality in type 2 diabetes. 
N Engl J Med 2015;373:2117-28.

67. Kohler S, Kaspers S, Salsali A, et al. Analysis of fractures in patients with type 2 diabetes treated with empagliflozin in pooled data from placebo-controlled trials and a head-to-head study versus glimepiride. Diabetes Care 2018;41:1809-16.

68. Ruanpeng D, Ungprasert P, Sangtian J, et al. Sodiumglucose cotransporter 2 (SGLT2) inhibitors and fracture risk in patients with type 2 diabetes mellitus: A metaanalysis. Diabetes Metab Res Rev 2017;33. doi: 10.1002/ dmrr.2903.

69. Thrailkill KM, Lumpkin CK Jr, Bunn RC, et al. Is insulin an anabolic agent in bone? Dissecting the diabetic bone for clues. Am J Physiol Endocrinol Metab 2005;289:E735-45.

70. Xi G, Rosen C, Clemmons D. IGF-I and IGFBP-2 stimulate AMPK activation and autophagy, which are required for osteoblast differentiation. Endocrinology 2016;157:268-81.

71. Nyman JS, Kalaitzoglou E, Clay Bunn R, et al. Preserving and restoring bone with continuous insulin infusion therapy in a mouse model of type 1 diabetes. Bone Rep 2017;7:1-8.

72. de Waard EAC, Driessen JHM, de Jong JJA, et al. The association between insulin use and volumetric bone

Cite this article as: Guja C, Guja L, Miulescu RD. Effect of type 2 diabetes medications on fracture risk. Ann Transl Med 2019;7(20):580. doi: 10.21037/atm.2019.09.51 mineral density, bone micro-architecture and bone strength of the distal radius in patients with type 2 diabetes - The Maastricht study. Bone 2017;101:156-61.

73. Schwartz AV, Sellmeyer DE, Ensrud KE, et al. Older women with diabetes have an increased risk of fracture: a prospective study. J Clin Endocrinol Metab 2001;86:32-8.

74. Moayeri A, Mohamadpour M, Mousavi SF, et al. Fracture risk in patients with type 2 diabetes mellitus and possible risk factors: a systematic review and meta-analysis. Ther Clin Risk Manag 2017;13:455-68.

75. Khunti K, Damci T, Meneghini L, et al. Study of Once Daily Levemir (SOLVE ${ }^{\mathrm{TM}}$ ): insights into the timing of insulin initiation in people with poorly controlled type 2 diabetes in routine clinical practice. Diabetes Obes Metab 2012;14:654-61.

76. Wallander M, Axelsson K, Nilsson A, et al. Type 2 diabetes and risk of hip fractures and non-skeletal fall injuries in the elderly: a study from the fractures and fall injuries in the elderly cohort (FRAILCO). J Bone Miner Res 2017;32:449-60.

77. Pscherer S, Kostev K, Dippel FW, et al. Fracture risk in patients with type 2 diabetes under different antidiabetic treatment regimens: a retrospective database analysis in primary care. Diabetes Metab Syndr Obes 2016;9:17-23. 